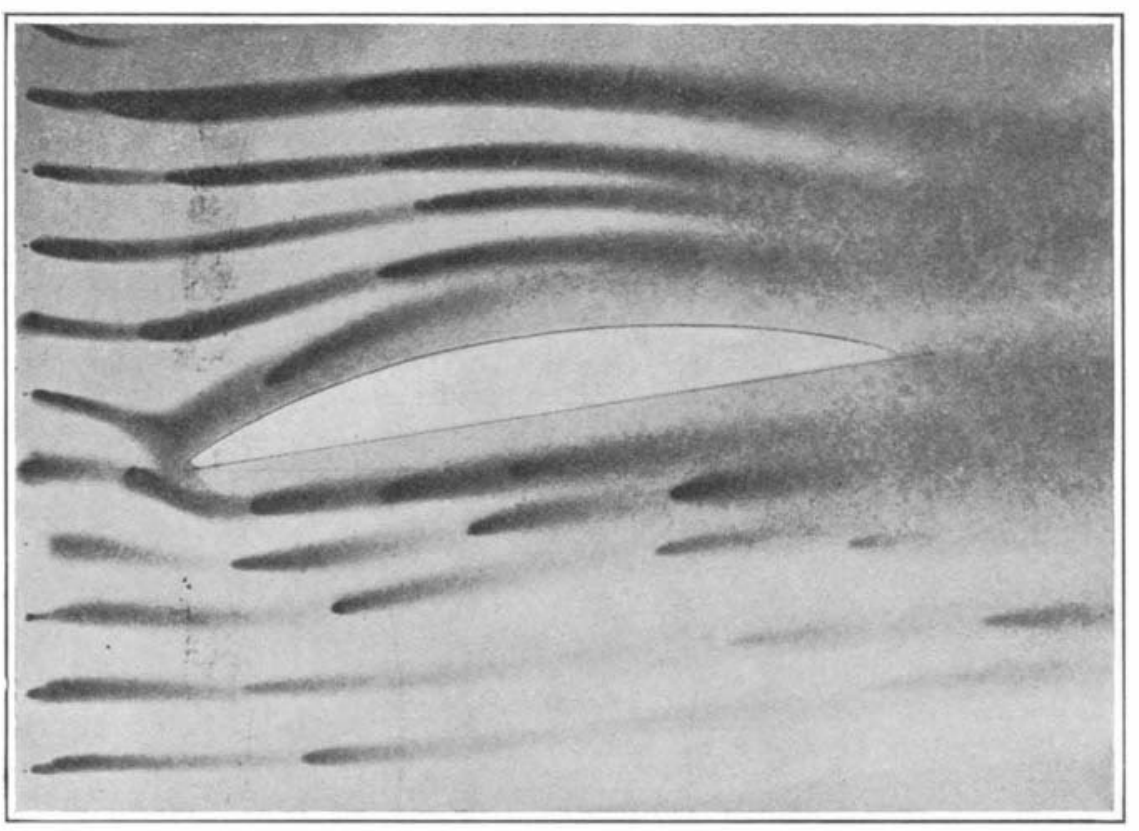

Fig. 5.-Stream Lines of Air About a Circular Segment Inclined to the Direction of the Wind. Speed, 220 Feet per Second.

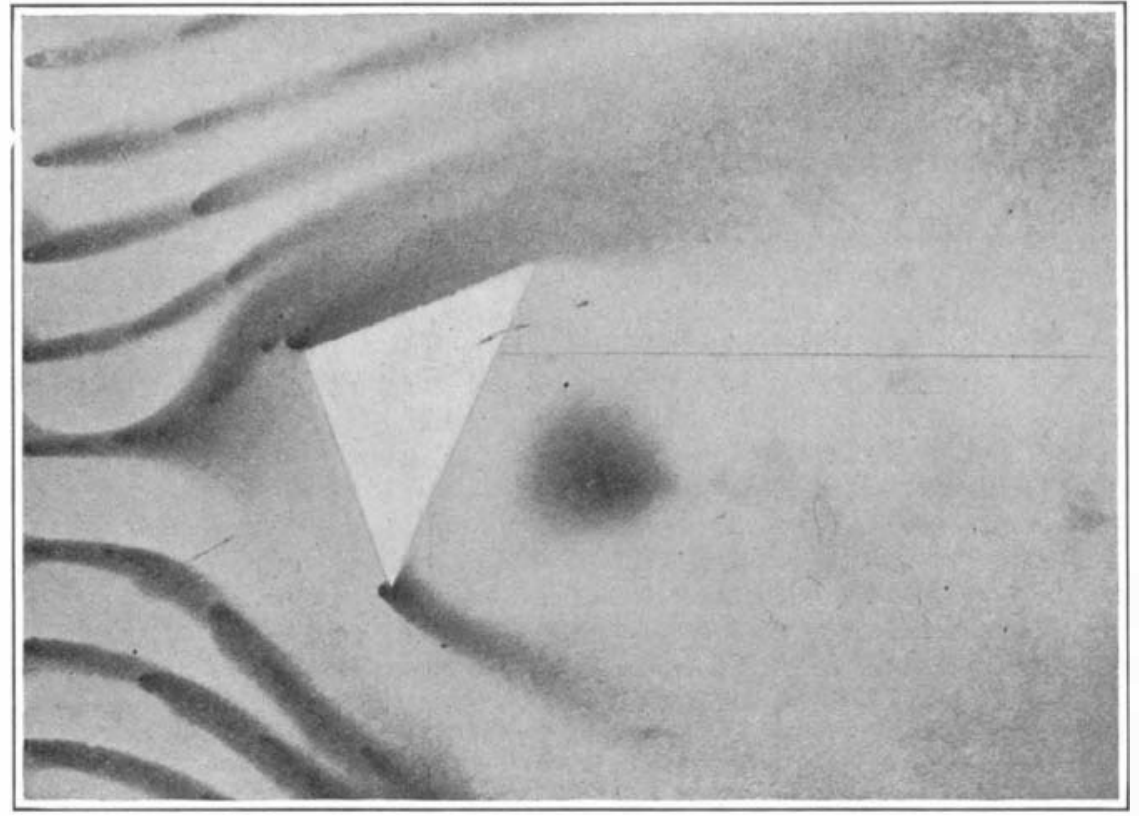

Fig. 6.-Stream Lines Around Triangular Prism. Note the Dead Zøne Behind the Prism.
$4 / 100$ of an inch in diameter, and placed $16 / 100$ of an projected and caused t• fiow along over the surface of the plate covered with prepared paper. These streams of gas are carried along by the main air current, and made t• pass •ver the plate and there produce dark streaks. The efrect is shøwn, for example, in Fig. 1, in which the gases fiow from left to right. The obstacle placed in the path of the air current was shaped like the segment of a circle and was inclined at about 12 degrees to the current, which had a velocity of 220 feet per second. The result thus obtained was not entirely satisfactory, the gas gradually diffusing as it passes along, so that the record becomes hazy and finally obliterated before the back edge of the obstacle is reached. But after repeated efforts, Mr. Chichloff finally found the means to overcome these drawbacks and obtained very excellent records. Our illustration, Fig. 3, shows a view of the wind box which is supplied with air from a suitable source and has an opening in front from which a practically uniform air current is

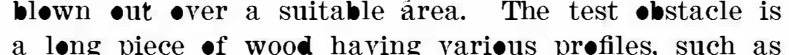
a segment of a circle, a triangle, etc. This is placed across the mouth of the box. Attached to the obstacle is the thin metal plate covere with paper as described above. A stream of air is bubbled through a bottle of ammonia, sø that it carries with it a charge of ammonia gas. It then passes through a rubber tube to a small glass tube ending in a very fine point. This tube is held perpendicular to the test plate and has a single small hole of $4 / 100$ of an inch in diameter. Through this a very fine gas stream is delivered on the plate. With a little practice it is n॰t difficult t $\bullet$ the correct position in which to hold the tube so as to get the best possible effect. Using this arrangement and møving the gas jet from point to pøint, wherever a record was to be made, Mr. Chichkoff succeeded in obtaining exceedingly clear records of the air movement in the space surrounding the test obstacle. The method followed is first of all to mark with a pencil a number of pöints in frønt of the obstacle, as starting pøint frøm which to begin one's observations. These pøints will be seen marked on the extreme left of Fig. 2. A tube is then placed opposite each of these points in turn, and a streak of about $1 / 2$ inch in length is obtained. The course of this streak is then followed up by hand with the tube, and so $\bullet$, until a complete set of fiow lines is obtained. Instead of studying the conditions in front and around the obstacle, it is of course equally possible to make observations at the rear and to obtain a representation of the vortex movement in what is known as the dead zone. Images obtained here are shown in Fig. 4, the obstacle in this case being a prism of triangular cross section. The prism is placed in two different nositions in the air current, with a wind speed of 35 meters ( 250 feet) per second. It is best in this case to introduce the gas directly into the dead zone in order t• obtain a clear image.

Mr. Chichkoff's results represent a very valuable contribution to the general study of air movement, and his method has the great advantage of being very easily carried out and furnishing a permanent record.

\section{Conduction of Electricity Through an Insulator} By P. S. Helmick

FEw persons think of the flow of an electric curren without picturing some metallic medium, as a lamp-cor or a telephone wire, through which it may pass.

It is the purpose of these notes to supplement that view, by illustrating how conduction may, as proved by the results of several experiments, take place across medium usually classified as a dielectric, and through which no ordinary electric current can flow.

Air is usually regarded as an excellent insulator, but every one has seen a spark leap across an air-gap, under the influence of an electromotive force of several thousand volts. This phenomenon is usually explained, by assuming that there are always a few ions, or "carriers of electricity," which, under the action of an electric current are imparted a higher velocity, and are thus enabled to break down the mol until there are enough present to carry a current, which is really the spark.

is really the spark.
In the Philosophical Magazine of August, 1912, Prof. R. W. Wood describes methods whereby he was able to detect conduction through dielectrics. He "half-silvered" thin quartz plates, and then, by successive rulings and cross-rulings, broke up this thin deposit of silver into small squares with sides of 0.001 centimeter length Yet the electrical resistance after ruling, was practically the same as before, although the cuts extended cleanly the same as before, although the cuts extended cleanly and distinctly through the silver. Of course, conduc-
tion must have taken place, to account for the constancy

of the resistance.

He then deposited a film of 0.3 centimeter wide, havin a resistance of $10 \mathrm{ohms}$, upon a glass plate, and after making 20 diamond cuts, of width 0.00003 centimeter across it, the resistance had increased but $3 \mathrm{ohms}$. Conduction had taken place at the low potential of 0.001 volt With cuts 0.00006 centimeter in width, however, no trace of conduction was found.

Prof. Wood suggests that possibly the conductivity at the above low voltages might possibly be explained by the "discharge from points" phenomenon, for the minute rregularities upon the edge of the metallic deposit may be assumed to act as points.

He accordingly began experiments with "optical flats" of highly-polished, glass-hard, speculum metal which deviated from true planes by less than 0.0000015 centimeter. When the plates were separated by two quartz fibers, each some 0.000015 centimeter in diameter, a potential of 1 volt produced a current of several milliamperes. The current increased rapidly and steadily as pressure was applied to the planes, although it returned to exactly its original value when the force was removed. to exactly its original value when the force was removed.
Conduction was also observed with finely powdered sulphur, with quartz dust, and with lycopodium spores. With small flakes of extremely thin mica, conduction took place in the neighborhood of 0.00075 centimeter under a potential of 1 volt

Prof. Wood concludes that the effect is caused by the "presence of an atmosphere of electrons in the immediate proximity of metallic surfaces."

Following out the experiments and suggestions made by Prof. F. C. Brown, Mr. H. H Blanchard and I were enabled to investigate along the same lines, using two brass cylinders, each 0.6 centimeter in diameter and having one perfectly plane base. The true surface of one of the cylinders was dipped in the finely sifted dielectri material with which the eylinders are to be kept from metallic contact-in this case sulphur and precipitated selenium - the majority of the minute particles blown off by means of compressed air, and the other cylinder set gently on top of the first.

A Dolezalek quadrant electrometer, having a capacity A 80 centimeters, was then charged to a potential of 4 volts and connected in parallel with the cylinders. The latter, having a capacity by virtue of the small film of dielectric and air, receives some of the charge from the electrometer, so that its reading is diminished-just as any charge distributes itself over two bodies which are in electrical contact. It is then possible to obtain the distance separating the plates from the respective reading of the electroct and the cross-sectional area of the cylinders Let $C_{1}$ be the capacity of the electrometer
a

$C_{2}$ the capacity of the cylinders and air-gap,

\begin{tabular}{|c|c|c|c|c|c|c|c|}
\hline $\begin{array}{l}\text { Dielectric Used to Separate } \\
\text { Cylinders. }\end{array}$ & $\begin{array}{l}\text { Sample } \\
\text { No. }\end{array}$ & $\begin{array}{l}\text { Electrometer } \\
\text { Zero. }\end{array}$ & $\begin{array}{c}C_{1} \\
\text { Charged. }\end{array}$ & $\begin{array}{l}C_{1} \text { and } C_{2} \\
\text { Mixed. }\end{array}$ & $\begin{array}{c}l \text { by } \\
\text { formula, } \\
\text { centimeter. }\end{array}$ & \begin{tabular}{|l}
$\quad l$ by \\
microscope, \\
centimeter.
\end{tabular} & Remarks. \\
\hline Sulphur. & $\begin{array}{l}1 \\
1 \\
1 \\
2 \\
3 \\
3 \\
4 \\
\mathbf{4} \\
5 \\
5 \\
5 \\
6 \\
6\end{array}$ & $\begin{array}{l}220 . \\
228.5 \\
228 . \\
273 . \\
272 . \\
272.8 \\
270 . \\
270 . \\
271 . \\
270.5 \\
271 . \\
271 . \\
271 .\end{array}$ & $\begin{array}{l}283 . \\
282.5 \\
282 . \\
323.2 \\
324.8 \\
325.3 \\
322 . \\
321 . \\
321 . \\
321 . \\
322 . \\
322 . \\
323 .\end{array}$ & $\begin{array}{l}240 . \\
241.5 \\
245 . \\
273 . \\
303.0 \\
303.0 \\
270 . \\
275 . \\
273 . \\
270.5 \\
275.5 \\
271 . \\
296 .\end{array}$ & $\begin{array}{l}0.000131 \\
0.0000869 \\
0.000129 \\
0.0000000 \\
0.00040 \\
0.00038 \\
0.000000 \\
0.000039 \\
0.000013 \\
0.000000 \\
0.0000218 \\
0.000000 \\
0.000261\end{array}$ & $\begin{array}{c}\ldots \ldots \\
0.00015 \\
0.0012 \\
0.00027 \\
\cdots \cdots \\
\cdots \cdots \\
\cdots \cdots \\
0.00015\end{array}$ & $\begin{array}{l}\text { No leakage. } \\
\text { slow leakage. } \\
\text { Very slow leakage. } \\
\text { Conducting. } \\
\ldots \ldots . . \\
\text { Conducting... } \\
\text { No leakag. } \\
\text { No leakage. } \\
\text { Conducting. } \\
\text { Conducting... } \\
\text { No leakage. }\end{array}$ \\
\hline
\end{tabular}

$d_{1}$ the reading of the charged electrometer

$d_{2}$ the reading of the electrometer after $C_{1}$ is connected to $C_{2}$, and $l$ the distance separating the cylinders whose cross-sectional area $=A$.

As $d_{1}$ and $d_{2}$ are comparatively small, $C_{2}=C_{1}\left(d_{1}-d_{2}\right)$

But $C_{2}=\frac{A k}{4 \pi l}$,

Combining, $l=\frac{A}{4 \pi d_{1}\left(d_{1}-d_{2}\right)}$

As a check method the particles were also measured by obtained

Below are the results of our experiments.

The above data indicate that with a potential difference of 4 volts, the maximum distance in ordinary air that the cylinders can be separated, in order that conduction may take place, is in the neighborhood of 0.0015 centimeters, as proved by the results in using both selenium and sulphur to separate the cylinders.

Now to explain the apparent inconsistency between the observed and the calculated value of $l$. The observed value with the micrometer eye-piece means that the cylinders are, as far as our human senses are concerned, that distance apart, while the calculated value indicates that they act as though situated from thirty to one hundred times nearer. This means that just outside the plane of the cylinders there must be located a carry the charge from one cylinder to the other.

From these results then it appears advisable, in order to explain this kind of conduction with the most facility: to accept Prof. Wood's hypothesis and assume that conduction may easily take place across two metallic electrodes saction

lies within a certain constant limit. means of a micrometer eye-piece and a mean value of $l$ swarm of conducting particles, or electrons, which can 